\title{
In vitro Propagation, Development and Validation of HPTLC Method for Quantification of Steroidal Glycoalkaloid Solasodine In vitro Culture and Plant Parts of Solanum nigrum
}

\author{
MOHD AMIR ${ }^{1}$, NIYAZ AHMAD², MOHAMMAD SARAFROZ ${ }^{3}$, MAHVISH JAMAL ${ }^{4}$, \\ FAHEEM HYDER POTTOO ${ }^{5}$ and MOHD MUJEEB ${ }^{4 *}$
}

\author{
${ }^{1}$ Department of Natural Product \& Alternative Medicines College of Clinical Pharmacy, \\ Imam Abdulrahman Bin Faisal University, Dammam, 1982, Saudi Arabia. \\ 2Department of Pharmaceutics, College of Clinical Pharmacy Imam Abdulrahman Bin \\ Faisal University, Dammam, 1982, Saudi Arabia. \\ ${ }^{3}$ Department of Pharmaceutical Chemistry College of Clinical Pharmacy Imam \\ Abdulrahman Bin Faisal University, Dammam, 1982, Saudi Arabia. \\ ${ }^{4}$ Department of Pharmacognosy and Phytochemistry Faculty of Pharmacy \\ Jamia Hamdard, New Delhi-62, India. \\ ${ }^{5}$ Department of Pharmacology College of Clinical Pharmacy Imam Abdulrahman Bin \\ Faisal University, Dammam, 1982, Saudi Arabia. \\ ${ }^{*}$ Corresponding author E-mail: drmmujeeb12@ gmail.com \\ http://dx.doi.org/10.13005/ojc/340606
}

Received: January 31, 2018; Accepted: November 20, 2018)

\begin{abstract}
Solanum nigrum Linn. (Family: solanaceae), has medicinal values, commonly exploited for its active constituents which are of high pharmaceutical importance. The plant contains different type of natural compounds, and one of most important glycoalkaloid in S. nigrum is solasodine. Nodal segment of $S$. nigrum was cultured on MS media added with several concentrations of 2,4-dichlorophenoxy acetic acid (2, 4-D) (0.5 ppm-2 ppm) for callus induction and development. For shoot induction, MS medium complemented with different dilutions of 6-benzyl adenine (6-BA) $(0.5 \mathrm{ppm}-2 \mathrm{ppm})$ was used. A novel, rapid, and simple high performance thin layer chromatographic method for quantitative estimation of triterpenoid glycoalkaloid. Solasodine was spotted on silica gel $60 \mathrm{~F}_{254}$ TLC plate using chloroform: methanol (8.75: 1.25; v/v) as mobile phase. Anisaldehyde-sulfuric acid in methanol used as derivatizing reagent and quantified by densitometric study at $366 \mathrm{~nm}$. This scheme was found to give a sharp and compact spot for the standard drug $\left(R_{f}=0.67 \pm 0.04\right)$. The linearity of this method was found to be a range of 100-1000 ng per spot with LOD and LOQ of 23.78 ng per spot and $67.09 \mathrm{ng}$ per spot; respectively. This method has shown recoveries of solasodine in the range from $98.23 \%-100.88 \%$, with low intra and inter-day \% RSD. Statistical investigation of the data presented that the method is selective, accurate, precise and reproducible for the study of solasodine. The established and developed HPTLC method would be a significant parameter for the analysis of quality control method of herbal drugs and formulations.
\end{abstract}

Keywords: Solanum nigrum, solasodine, Glycoalkaloid, HPTLC.

This is an Open Access article licensed under a Creative Commons license: Attribution 4.0 International (CC- BY). Published by Oriental Scientific Publishing Company @ 2018 


\section{INTRODUCTION}

Medicinal natural herbs are used by $80 \%$ of the global population as the only existing medicines exclusively in emerging countries ${ }^{1}$. The consumption of plant cells and tissues for the production of natural constituents of marke table attention has grown accumulative attention over past few decades ${ }^{2}$. Currently, there is a great reduction in herbal resources due to human disturbances of the natural atmosphere. Plant cell and tissue cultures hold prodigious promise for controlled manufacture of myriad of valuable secondary metabolites on demand ${ }^{3}$. Therefore, biotechnology hopes for a bypass to overwhelmed this difficult by using cell culture technique and advance multiplication of significant herbal drugs by micropropagation technique. Now, the technique of tissue culture has been established for great scale cultivation of natural medicine. The production of valuable metabolites from plant tissue culture has produced a new procedure for their commercialization ${ }^{4}$.

Solanum nigrum L. (Black night shade) is belongs to family Solanaceae, has an extensivevariety of the rapeutic values. The plant has antiseptic, antidysenteric and antidiuretic activity; used in the treatment of skin disease, cardiac disease, psoriasis, herpsvirus and inflammation of kidney ${ }^{5}$. Plant such as $S$. nigrum contains glycoalkaloids as the main phytoconstituents. Glycoalkaloids concentration depends upon species as well as part of plant such as leaf, root, flower and stem. The amount of glycoalkaloids increases due to injury and exposure to light ${ }^{6}$. Glycoalkaloids play key role in defense mechanism of plants against invading microbes. Glycoalkaloids are used in insecticidal, antimicrobialand fungicidal action which account for their activity against numerous insects, pests and herbivores ${ }^{7}$. Solasodine (Fig. 1) is the active

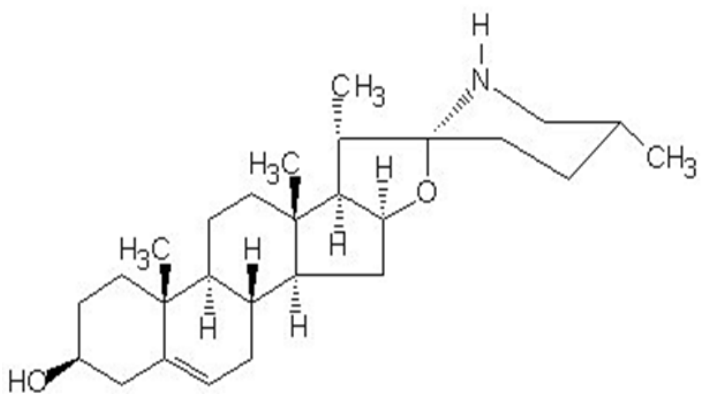

Fig. 1. Structure of solasodine constituent of S. nigrum, a steroidal glycoalkaloid, an $\mathrm{N}$-analogue of diosgenin ${ }^{8}$, which have its immense use as a precursor for commercial synthesis of steroidal medicines and has got valuable scope and demand in pharmaceutical industry. Solasodine is obtained by chemical or microbial hydrolysis of solamargine ${ }^{9}$.

There is a dearth of analytical methods of solasodine as a chemical and biological marker for quality control of this important medicinal plant. Hence, in this study, it has also been proposed to establish an economic, simple, easy and fast analytical HPTLC method, which was validated as per the $\mathrm{ICH}$ guidelines and several other methods reported by laboratory ${ }^{10-11}$. The projected method for analysis of solasodine was applied in plant part and in In vitro callus cultures of $S$. nigrum, which proved that the scheme can be applied for quality control of this medicinal plant along with for traditional Ayurvedic and Unani formulations having it as a constituent. The objective of the current work, to make the callus formation and development from standard size of explants from young tissues and the comparative estimation of solasodine from field developed plant parts and In vitro callus culture.

\section{MATERIAL AND METHODS}

\section{Procurement and identification of plant part}

The plant part was gathered from the plant developing in Hamdard university Herbal Garden, New Delhi and was authenticated by taxonomist at Faculty of Science, Hamdard University.

\section{Chemicals and reagents}

Solasodine (98.50\%) was bought by Sigma Aldrich, USA. Each chemical and reagent were of at least analytical grade. Aluminum-backed TLC plates pre-coated with $0.2 \mathrm{~mm}$ layer of silica gel 60 F254 (20 $\mathrm{cm} \times 10 \mathrm{~cm}$ ) were obtained from E. Merck, Germany; supplied by Anchrom Technologists, Mumbai.

\section{In vitro propagation \\ Surface sterilization}

The explants were washed carefully with $2 \%$ Tween-20 followed by tap water for $10-15 \mathrm{~min}$. and then again washed three time with double distilled water. The plant parts were then dipped in $70 \%$ ethanol for one minute, after that surface 
sterilization with $0.1 \%$ mercuric chloride for $10 \mathrm{~min}$. and finally rinsed thrice by sterile double distilled water.

\section{Murashige and Skoog basal medium}

The plant parts were surface dried on sterile mesh paper and transferred on Murashige and Skoog (MS) basal media complemented by various dilutions and combinations of plant growth hormones. The culture media contained of MS medium comprising basal salts and vitamins with $3 \%(\mathrm{w} / \mathrm{v})$ sucrose, $0.8 \%$ agar and different growth regulators. The $\mathrm{pH}$ of all cultures medium was changed in accordance with 5.8 before autoclaving at $121^{\circ} \mathrm{C}$ for 20 minutes $^{12}$.

\section{Callus induction and development}

For the initiation, development and maintenance of static callus, sterilized explant was transferred aseptically on MS medium added with various concentrations of $2,4-D(0.5 \mathrm{ppm}-2$ $\mathrm{ppm})$. All the cultures were incubating at $25 \pm 2^{\circ} \mathrm{C}$ under $16 \mathrm{~h}$ photoperiod supplied in BOD incubator for callus initiation and development ${ }^{13-15}$. The calli were subcultured in MS media complemented with the same concentration of plant growth regulator in every 4 weeks. All calluses was separated into $2-4$ $\mathrm{mm}$ diameter pieces during transfer and subcultured up to eight times for best result.

\section{Micropropagation}

The sterilized explant was transferred aseptically on MS medium supplemented with various dilution of plant growth regulator for micropropagation. For shoot regeneration from nodal segment, MS medium supplemented with various concentrations of 6 - BA ( $0.5 \mathrm{ppm}-2 \mathrm{ppm})$ was used. Each cultures were incubating at $25 \pm 2^{\circ} \mathrm{C}$ under $16 \mathrm{~h}$ photoperiod supplied in BOD incubator for one month for shoot regeneration ${ }^{13-15}$. The plantlets were subcultured in MS medium supplemented with the same dilution of plant growth regulator in every 4 weeks. Each plantlet were divided into 2-4 pieces during transfer and subcultured up to eight times for best result.

\section{Analysis of Solasodine in S. nigrum by HPTLC Standard preparation}

A standard solution of solasodine was ready by dissolving $10 \mathrm{mg}$ of accurately weighed solasodine in $10 \mathrm{ml}$ methanol in a standard volumetric flask. The final concentration of stock solution was $1 \mathrm{mg} / \mathrm{ml}$. Different dilutions of standard solasodine were prepared from stock solutions and filtered through $0.2 \mu \mathrm{m}$ membrane filter (Axiva) and then subjected to HPTLC analysis.

\section{Preparation of test samples}

Weigh powdered of callus and plant part of $S$. nigrum ( $2 \mathrm{~g}$ each) and were macerated in $20 \mathrm{~mL}$ methanol $(3 \times 120 \mathrm{~min}$. using magnetic stirrer to ensure complete extraction). The filtered methanolic extract from all the two extracts were concentrated under reduced pressure. Both sample solutions were then filtered through $0.2 \mu \mathrm{m}$ membrane filter and subjected to HPTLC analysis.

\section{Chromatographic procedure}

The tests were spotted in the form of bands ( $5 \mathrm{~mm}$ width) with a syringe (Camag Microliter) under controlled nitrogen stream on silica gel (precoated) aluminium plate $60 \mathrm{~F}(20 \mathrm{~cm} \times 10 \mathrm{~cm}, 200 \mu \mathrm{m}$ thickness, Merck, Darmstadt, Germany) using sample applicator (Camag Linomat V, Switzerland). Space between two bands was maintained $11.6 \mathrm{~mm}$ with slit dimension at $4 \mathrm{~mm} \times 0.45 \mathrm{~mm}$, and scanning speed at $20 \mathrm{~mm} / \mathrm{s}$. The mobile phase was composed of chloroform: methanol (8.75: 1.25; v/v). Camag twin trough glass chamber saturated with the mobile phase for $30 \mathrm{~min}$. at $25 \pm 5^{\circ} \mathrm{C}$ with relative humidity of $60 \pm 5 \%$, was used for method development and the chromatogram was developed up to the length of $80 \mathrm{~mm}$. The TLC plates were then dried in an air dryer. Scanning for densitometric analysis was achieved by using TLC scanner III (CAMAG) operated using winCATS software at $366 \mathrm{~nm}$ after spraying with anisaldehyde-sulfuric acid (in absolute alcohol) reagent and drying in hot air oven at $110^{\circ} \mathrm{C}$ for 10 minutes. The source of radiation used was deuterium and tungsten lamp ${ }^{16}$.

\section{Method Validation}

The Proposed method was validated as per $\mathrm{ICH}$ guidelines ${ }^{10-11}$.

\section{Linearity (calibration curve)}

Standard solutions of solasodine were prepared in methanol to get a concentration of 1 $\mathrm{mg} / \mathrm{mL}$. Different volumes of standard solution were applied on the TLC plate in triplicate to gain a final dilution of 100-1000 ng per spot for solasodine. The plate was then developed and the data of peak 
area/height versus drug concentration were treated by linear least squares regression analysis to get a regression equation.

\section{Accuracy}

The method accuracy was analyzed by calculating recoveries of solasodine using standard addition method. Standard solutions of solasodine known quantities were added to pre-quantified sample solution of solasodine at 50,100 and $150 \%$ level. The quantity of solasodine was assessed by applying attained values to the respective regression line equations.

\section{Precision}

The intra-day and inter-day precision for the estimation of solasodine was carried out at two various dilution levels of 600 and $800 \mathrm{ng}$ per spot. The precision of the system and method were expressed as \%RSD and the SD of peak area/height.

\section{Robustness, LOD and LOQ}

The method robustness was determined by introducing minor variations in the composition of the mobile phase. Variations in the composition of chloroform: methanol was tried at two different concentration levels of 600 and 800 ng per spot and $\%$ RSD of peak area/height was observed. LOD was expressed as $(3.3 \times \sigma) /($ slope of calibration plot) and LOQ was expressed as $(10 \times \sigma) /($ slope of calibration plot). Blank methanol was applied $n=6$ times and the $\mathrm{SD}$ of the analytical response were analyzed.

Estimation of solasodine in callus and plant part of $S$. nigrum $2 \mu$ of each samples as prepared above was applied in triplicates on TLC plates. It was developed and scanned as per the method described. The results of peak area/height obtained corresponding to solasodine were used for quantification in samples using regression equation. The results of triplicate analysis were expressed as average amount of solasodine in \%w/w.

\section{RESULT AND DISCUSSION}

\section{In vitro propagation}

To minimize the contamination caused by fungus, endogen and exogen bacteria, the explants of S. nigrum was subjected to treatment with different chemical sterilants for their surface sterilization.
When the plant part of $S$. nigrum was cultured on MS medium supplemented with 2, 4-D (0.5ppm) was found to be the best induction and development of callus. Callus growth was initiated in one week and fast growth followed for majority of cultures. The calli were soft, friable, and whitish brown in colour (Fig. 2a). In our study it was observed that the maintained callus was found to be compact and brownish black in colour it may be due to the accumulation of phenolic compounds. This phytohormone was further used for callus development and maintenance.MS medium supplemented with $6 \mathrm{BA}$ (1ppm) was found to be the best induction and development of shoots (Fig. 2b). Shoot buds initiated were light green to yellowish in color and were formed either as single or in clusters. This growth regulator was further used for shoot development and maintenance.

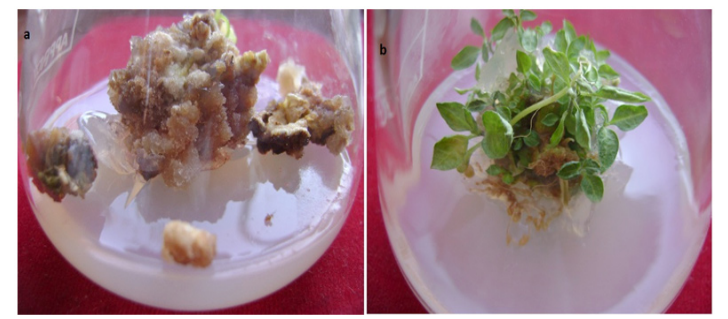

Fig. 2. (a) Development of callus culture; (b) Development of shoot formation

\section{Selection and Optimization of Mobile Phase}

The HPTLC method was developed and optimized. Numerous easily available solvents in various concentrations and combinations were tried to obtain good resolution, compact spot and better separation. The mixture of chloroform: methanol (8.75: 1.25; $\mathrm{v} / \mathrm{v}$ ) was confirmed to be better than the other mixtures in terms of resolution and peak shape. In this mobile phase the Rf value of solasodine was found to be 0.67 (Fig. 3) at $366 \mathrm{~nm}$.

\section{Validation of the Method}

The linearity of an analytical method is its capability to reproduce test results that are directly, exact mathematical modification and relative to the analyte concentration in drug samples within a precise range. It is also important that the elementary calibration plot be obtained by using independent samples, and not by using samples that have been prepared by dilution and spotted on TLC plate $^{17}$. Linear regression data for standard plot for solasodine $(n=3)$ was indicative of a good linearity 
(correlation coefficient, $\mathrm{r}^{2}=0.981$ and 0.980 ) between peak area and height in the series 100-1000 ng per spot (Table 1).Accuracy expresses the closeness of covenant between the value which is accepted as a predictable true value or an accepted reference value and the result found. This test allows the

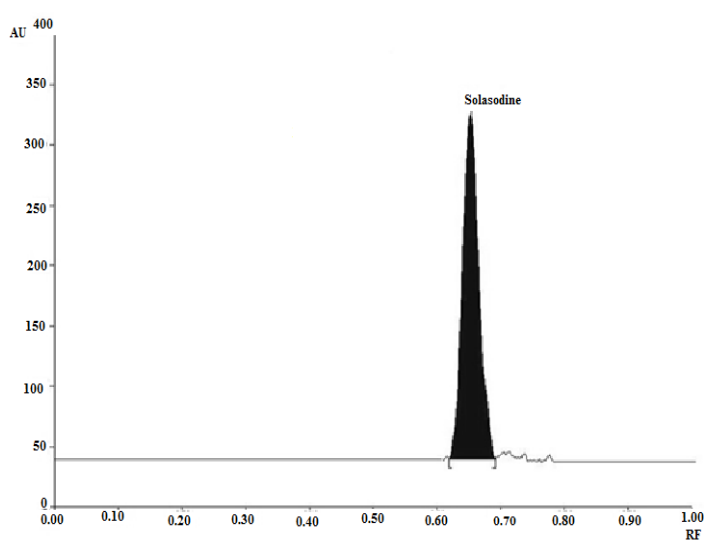

Fig. 3. A typical chromatogram of solasodine $\left(R_{f}=0.67\right)$ determination of possible bias ${ }^{18}$. The developed TLC method was used for extraction and consequent estimation of solasodine from S. nigrum after spiking with 50,100 and $150 \%$ of additional drug demonstrated good recovery of $98.23-100.88 \%$ for solasodine is summarized in Table 2.

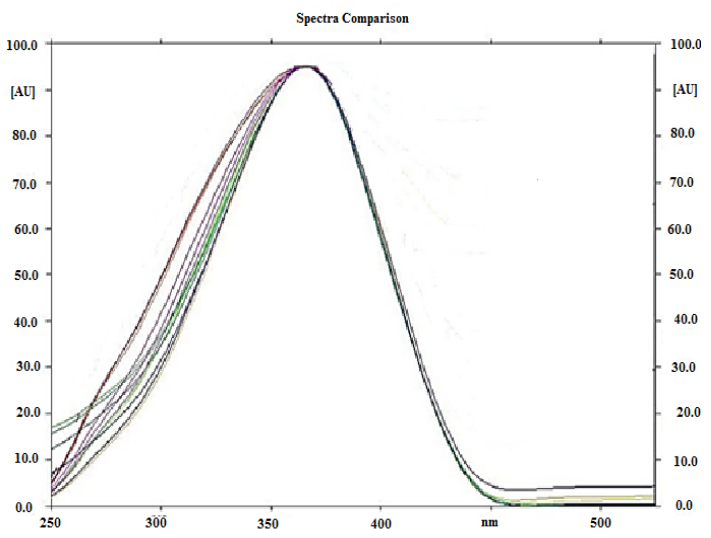

Fig. 4. Superimposed UV spectrum of solasodine in samples and standard $(n=3)$

Table 1: Linear regression data for the calibration plot $(n=3)$ for solasodine

\begin{tabular}{|c|c|c|c|c|c|}
\hline \multirow{2}{*}{\multicolumn{2}{|c|}{$\begin{array}{l}\text { Solasodine Linearity range }(\mathrm{ng}) \\
\text { Corr. Coeff. }\end{array}$}} & \multicolumn{2}{|c|}{ Slope } & \multicolumn{2}{|c|}{ Intercept } \\
\hline & & \pm SEM & Confidence limita & Mean \pm SEM & Confidence limita \\
\hline & 0 & & & 1 & 1 \\
\hline leight & $100-10000.980$ & $2.120 \pm 0.0446$ & $2.021-1.304$ & $108.13 \pm 3.05$ & $93.11-99.25$ \\
\hline
\end{tabular}

a95\% Confidence limit

Table 2: Recovery studies $(n=6)$, for solasodine

\begin{tabular}{cccccccc}
\hline Solasodine & \multicolumn{3}{c}{ Recovery (\%) } & \% RSD & \multicolumn{3}{c}{ SEM } \\
\hline $\begin{array}{l}\text { Excess drug added } \\
\text { to the analyte(\%) }\end{array}$ & $\begin{array}{c}\text { Theoretical } \\
\text { content }(\mathrm{ng})\end{array}$ & Height & Area & Height & Area & Height & Area \\
\hline 0 & 400 & 98.23 & 98.26 & 0.561 & 0.513 & 1.201 & 1.601 \\
50 & 600 & 98.72 & 99.16 & 0.554 & 0.445 & 1.025 & 1.464 \\
100 & 800 & 99.80 & 100.88 & 0.597 & 0.333 & 2.023 & 1.111 \\
150 & 1000 & 100.44 & 99.53 & 0.392 & 0.365 & 1.201 & 1.836 \\
\hline
\end{tabular}

The precision of an analytical procedure is measured by the quantity of covenant among individual test results from repeated analyses of a same sample. Precision is usually done at three various levels, namely repeatability, intermediate precision, and reproducibility ${ }^{19}$. Intra-day and interday precisions communicated in terms of \%RSD, which showing intra-day and inter-day difference of solasodine at two various concentrations of 600 and $800 \mathrm{ng} / \mathrm{sp}$ ot. The low \%RSD value (0.146-1.892\%) for solasodine obtained, indicated that the method is precise, is shown in Table 3 . The robustness of an analytical process is explained as a degree of its ability to stay diffident by minor but considered deviations in experimental parameters, giving a sign of the analytical method's aptness and consistency during normal use ${ }^{20}$. The values of $\% R S D$ for solasodine (0.104-1.828\%) as obtained subsequent to bringing deliberate variations in compositions of the mobile phase specified robustness of the method as shown in Table 4. No significant difference was observed in the slope values (ANOVA; P>0.05). The 
LOD is well-defined as the minimum quantity of a drug in a sample that can be detected, but not really quantified. It is a limit test that determines whether an analyte is above or underneath a specific value. The LOQ in an another way in a sample can be quantified with reasonable precision and accuracy under the expressed functioning states of the method ${ }^{21}$.
Signal-to-noise ratios of 3: 1 and 10: 1 were found for the LOD (23.78 ng/spot) and LOQ 67.09 (ng/spot), respectively. The peak purity of standard solasodine and sample of solasodine showed same wave length at the peak start, top and peak end points of the spot when scanned at $366 \mathrm{~nm}$.

Table 3: Intra-day and intera-day precision of HPTLC method $(n=6)$ for solasodine

\begin{tabular}{|c|c|c|c|c|c|c|c|c|}
\hline \multirow{2}{*}{$\begin{array}{l}\text { Solasodine } \\
\text { Amount (ng per spot) }\end{array}$} & \multirow{2}{*}{$\begin{array}{l}\text { Mean } \\
\text { Height }\end{array}$} & \multicolumn{3}{|c|}{ SD } & \multirow{2}{*}{$\begin{array}{l}\% \text { RSD } \\
\text { Height }\end{array}$} & \multicolumn{3}{|c|}{ SEM } \\
\hline & & Area & Height & Area & & Area & Height & Area \\
\hline \multicolumn{9}{|l|}{ a. Intra-day } \\
\hline 600 & 862.51 & 7624.88 & 2.112 & 2.823 & 0.987 & 0.413 & 0.749 & 1.123 \\
\hline 800 & 991.36 & 9583.61 & 2.214 & 1.938 & 0.683 & 0.743 & 0.698 & 1.021 \\
\hline \multicolumn{9}{|l|}{ b. Inter-day } \\
\hline 600 & 877.14 & 7690.26 & 2.912 & 3.816 & 1.754 & 0.263 & 0.918 & 1.781 \\
\hline 800 & 973.46 & 9334.66 & 2.261 & 2.829 & 1.212 & 0.146 & 0.910 & 1.892 \\
\hline
\end{tabular}

Table 4: Robustness of the method $(n=3)$ for solasodine

\begin{tabular}{lcccc}
\hline Solasodine & \multicolumn{4}{c}{ Mobile phase composition } \\
\hline \multirow{3}{*}{ Amount (ng per spot) } & \multicolumn{2}{c}{ chloroform: methanol } & Petroleum ether: Isopropanol \\
& $(8.50: 1.50 \mathrm{v} / \mathrm{v}) \% \mathrm{RSD}$ & $(8.25: 1.75 \mathrm{v} / \mathrm{v}) \%$ RSD \\
& Height & Area & Height & Area \\
\hline 600 & 1.828 & 0.104 & 1.658 & 0.881 \\
800 & 1.263 & 0.164 & 0.863 & 0.863 \\
\hline
\end{tabular}

Determination of solasodine in callus and plant part of S. nigrum

A well resolute single spot of solasodine was detected at $R_{f} 0.67$ in the chromatogram of samples extracted from callus and plant part of S. nigrum. It was also observed from the super imposed UV spectra that there was no interference from the other constituents existing in the extracts (Fig. 4). The solasodine content in the callus and powdered plant part in $S$. nigrum were found to be $234.76 \mathrm{mg} / \mathrm{L}$ and $153.91 \mathrm{mg} / \mathrm{L}$, respectively. The low $\% R S D$ value indicated the appropriateness of this method for routine study of solasodine in $S$. nigrum during the tissue culture studies of solasodine.

\section{CONCLUSION}

The present study is significant from the point of view of the fact that the solasodine was found to be in the callus which was extracted and quantitatively estimated for the first time. The content of solasodine was also found to be higher in the callus extract as compare to plant extract. HPTLC exhibits several advantages over HPLC and consequently it is sometimes favored over it. HPTLC consumes by far less amount of solvents and therefore can be regarded as more economic and environment friendly. In addition, it is a fast method of analysis allowing the simultaneous processing of large number of samples with no memory effect. Furthermore, it allows the detection of all compounds even those strongly retained on baseline. Indeed, HPTLC does not require elaborate treatment or the sophisticated experimental setup usually associated with HPLC methods of analysis. This work describes a simple, sensitive and robust HPTLC method for the simultaneous determination of the aforementioned drug in their plant part and callus. The proposed method met the $\mathrm{ICH}$ validation acceptance criteria concerning; linearity, robustness, precision and accuracy.

\section{ACKNOWLEDGEMENT}

The authors are thankful to the Jamia Hamdard, New Delhi-India and Imam Abdulrahman Bin Faisal University, Dammam-Kingdom Saudi Arabia for providing financial and technical assistance to carry out the research work. 


\section{REFERENCES}

1. Hashim, H.; Kamali, E.L.; Mohammed, Y Current Res J Biol Sci., 2010, 2, 143-146.

2. Canter, P.H.; Thomas, H.; Ernst, E. Trends Biotechnol., 2005, 23, 180-185.

3. Hussain, S.M.; Fareed, S.; Ansari, S.;Rahman, M.A.; Ahmad, I.Z.; Saeed, M. J Pharm Bioallied Sci., 2012, 4, 10-20.

4. Bolta, Ž.; Barǐcevǐc, D.; Bohanec, B.;Andrenšek, S. Plant Cell Tissue Org. Cult., 2000, 62, 63-75.

5. Indian Medicinal Plants [8 VOLUME SET] by Kirtikar, K. R.; Basu, B. D.: Bishen Singh Mahendra Pal Singh, Dehra Dun - The Book Gallery https://www.abebooks.com/IndianMedicinal-Plants-8-VOLUME-SET/8860864437/ bd (accessed Sep 16, 2018).

6. Arnqvist, L.; Dutta, P.C.; Jonsson, L.; Sitbon, F. Plant Physiol., 2003, 131, 1792-1799.

7. Kanika, P.; Ravi, B.S.; Dinesh, K.P. J Acute Dis., 2013, 92-98.

8. Mann, J. D. Production of Solasodine for the Pharmaceutical Industry. In Advances in Agronomy; Brady, N. C., Ed.; Academic Press., 1979, 30, 207-245.

9. Mann JB. Adv Agron., 1987, 30, 207- 254.

10. Validation of Analytical Procedures: Text and Methodology : ICH http://www.ich.org/products/ guidelines/quality/quality-single/article/validationof-analytical-procedures-text-and-methodology. html (accessed Sep 16, 2018).
11. Amir, M.;Mujeeb,M.; Ahmad, S.;Akhtar, M.; Ashraf, K. Pharm Methods., 2013, 4, 62-67.

12. Murashige T, Skoog F. Plant Physiol., 1962, 15, 473-479.

13. Nhut, D.T.; Duy, N.;Vy, N.N.H.;Khue, C.D.; Khiem, D.V.; Vinh, D. N. J of Applied Horticulture., 2006, 8, 135-137.

14. Te-chato, S.; Susanon, T.; Sontikun, Y. Songklanakarin., J. Sci. Technol., 2006, 28, 717-722.

15. Dodds, J. H.; Roberts, L. W. Experiments in Plant Tissue Culture, 3rd ed.; Cambridge [England] ; New York : Cambridge University Press, 1995.

16. Ashraf, K.; Mujeeb, M.; Altaf, A.;Ahmad, S.; Amir, M.; Khan, A.; Mallick, M.N.; Sharma, D. Asian Pac J Trop Biomed.2012,S584-S588.

17. Nash, R. A.; Wachter, A. H. Pharmaceutical Process Validation., 2003.

18. Ferenczi-Fodor, K.; Renger, B.; Végh, Z. J Planar Chrom., 2010, 23, 173-179.

19. Validation of Analytical Methods and Procedures http://www.labcompliance.com/ tutorial/methods/default.aspx (accessed Sep 16, 2018).

20. Shabir, G.A.; Lough, W.J.; Shafique, A.A.; Bradshaw, T.K. J Liq Chrom Rel Tech., 2007, 30, $311-333$.

21. Ferenczi-Fodor, K.; Renger, B.; Végh, Z. J Planar Chrom., 2010, 23, 173-179. 